\title{
COMPACT TOEPLITZ OPERATORS ON WEIGHTED HARMONIC BERGMAN SPACES
}

\author{
KAREL STROETHOFF
}

(Received 30 August 1996; revised 15 August 1997)

Communicated by A. H. Dooley

\begin{abstract}
We consider the Bergman spaces consisting of harmonic functions on the unit ball in $\mathbb{R}^{\prime \prime}$ that are squareintegrable with respect to radial weights. We will describe compactness for certain classes of Toeplitz operators on these harmonic Bergman spaces.
\end{abstract}

1991 Mathematics subject classification (Amer. Math. Soc.): 47B35, 47B38.

\section{Introduction}

Let $n \geq 2$ be a fixed integer. We use the notation $B=\left\{x \in \mathbb{R}^{n}:|x|<1\right\}$ and $S=\partial B=\left\{x \in \mathbb{R}^{n}:|x|=1\right\}$. Let $\sigma$ denote the rotation-invariant area measure on $S$, normalized so that $\sigma(S)=1$. If $u$ is harmonic on $B$, then $u$ has the mean value property

$$
u(y)=\int_{S} u(y+r \zeta) d \sigma(\zeta)
$$

whenever the ball $B(y, r) \subset B$. For $-1<\alpha<\infty$ let $d V_{\alpha}(x)=\left(1-|x|^{2}\right)^{\alpha} d V(x)$, where $V$ denotes Lebesgue volume measure on $\mathbb{R}^{n}$. The harmonic Bergman space $b^{2, \alpha}(B)$ is the space of all harmonic functions $u$ which are in $L^{2}\left(B, V_{\alpha}\right)$. Integrating (1.1) with respect to $r$ we obtain

$$
u(y)=\frac{1}{V_{\alpha}(B(y, r))} \int_{B(y, r)} u(x) d V_{\alpha}(x),
$$

The author was partially supported by grants from the Montana University System and the University of Montana.

(C) 1998 Australian Mathematical Society 0263-6115/98 \$A2.00+0.00 
whenever $B(y, r) \subset B$. Consequently, if $u$ is harmonic on $B$, then

$$
\begin{aligned}
|u(y)| & \leq \frac{1}{V_{\alpha}(B(y, r))^{1 / 2}}\left(\int_{B(y, r)}|u(x)|^{2} d V_{\alpha}(x)\right)^{1 / 2} \\
& \leq \frac{1}{V_{\alpha}(B(y, r))^{1 / 2}}\|u\| .
\end{aligned}
$$

whenever $B(y, r) \subset B$. Inequality (1.3) implies that $b^{2, \alpha}(B)$ is a closed subspace of $L^{2}\left(B, V_{\alpha}\right)$, thus a Hilbert space. We denote the orthogonal projection of $L^{2}\left(B, V_{\alpha}\right)$ onto $b^{2 . \alpha}(B)$ by $Q_{\alpha}$. For $f \in L^{\infty}(B)$ the Toeplitz operator $T_{f}: b^{2 . \alpha}(B) \rightarrow b^{2 . \alpha}(B)$ is defined by

$$
T_{f} u=Q_{\alpha}[f u], \quad u \in b^{2, \alpha}(B) .
$$

The operator $T_{f}$ is clearly bounded on $b^{2, \alpha}(B):\left\|T_{f}\right\| \leq\|f\|_{\infty}$. For certain classes of symbols we will describe when these operators are compact.

\section{Preliminaries}

Inequality (1.3) shows that for fixed $y \in B$ the linear functional $u \mapsto u(y)$ is bounded on $b^{2 . \alpha}(B)$. By the Riesz-Fischer Theorem, there exists a unique function $R_{\alpha}(\cdot, y) \in b^{2, \alpha}(B)$ for which

$$
u(y)=\left\langle u, R_{\alpha}(\cdot, y)\right\rangle, \quad u \in b^{2, \alpha}(B) .
$$

The mapping $R_{\alpha}$ is called the harmonic Bergman kernel of $b^{2 . \alpha}(B)$. Note that the projection operator $Q_{\alpha}$ is the integral operator with kernel $R_{\alpha}$ :

$$
Q_{\alpha}[f](y)=\int_{B} f(x) R_{\alpha}(x, y) d V_{\alpha}(x),
$$

for $f \in L^{2}\left(B, V_{\alpha}\right)$ and $y \in B$. The Bergman kernel is easily expressed in terms of the so-called zonal harmonics. We recall some terminology before we define these zonal harmonics.

A polynomial on $\mathbb{R}^{n}$ is homogeneous of degree $m$ (or $m$-homogeneous) if it is a finite linear combination of monomials $x_{1}^{k_{1}} \cdots x_{n}^{k_{n}}$, where $k_{1}, \cdots, k_{n}$ are non-negative integers such that $k_{1}+\cdots+k_{n}=m$. Note that a polynomial $p$ on $\mathbb{R}^{n}$ is homogeneous of degree $m$ if and only if $p(t x)=t^{m} p(x)$ for all $x \in \mathbb{R}^{n}$ and all $t \in \mathbb{R}$. The space $\mathscr{H}_{m}(S)$ of restrictions to $S$ of harmonic homogeneous polynomials of degree $m$, the so-called spherical harmonics of degree $m$, is a (finite-dimensional) Hilbert space with respect to the usual inner product on $L^{2}(S, d \sigma)$, where $\sigma$ denotes the normalized surface-area measure on $S$. For each $\xi \in S$ the linear functional $p \mapsto p(\xi)$ on 
the space $\mathscr{H}_{m}(S)$ is uniquely represented by a harmonic $m$-homogeneous polynomial $Z_{m}(\cdot, \xi)$, called the zonal harmonic of degree $m$ at $\xi$. Extending $Z_{m}$ to a function on $\mathbb{R}^{n} \times \mathbb{R}^{n}$ by setting $Z_{m}(x, y)=|y|^{m} Z_{m}(x, y /|y|)$, and using that each zonal harmonic $Z_{m}(\cdot, \xi)$ is real valued (see [1, pp. 78-79]) we have

$$
\int_{S} p(\zeta) Z_{m}(\zeta, y) d \sigma(\zeta)=p(y)
$$

for every harmonic $m$-homogeneous polynomial $p$. Spherical harmonics of distinct degrees are orthogonal, that is,

$$
\int_{S} p \bar{q} d \sigma=0
$$

if $p$ and $q$ are harmonic homogeneous polynomials of distinct degree. If $p$ is an $m$-homogeneous harmonic polynomial, then, using integration by polar-coordinates,

$$
\begin{aligned}
\int_{B} p(x) Z_{m}(x, y) d V_{\alpha}(x) & =n V(B) \int_{0}^{1} r^{n-1} \int_{S} p(r \zeta) Z_{m}(r \zeta, y) d \sigma(\zeta)\left(1-r^{2}\right)^{\alpha} d r \\
& =n V(B) p(y) \int_{0}^{1} r^{n+2 m-1}\left(1-r^{2}\right)^{\alpha} d r \\
& =\frac{n}{2} V(B) p(y) \frac{\Gamma\left(\frac{n}{2}+m\right) \Gamma(\alpha+1)}{\Gamma\left(\frac{n}{2}+m+\alpha+1\right)} .
\end{aligned}
$$

It follows that

$$
R_{\alpha}(x, y)=\frac{2}{n V(B)} \sum_{m=0}^{\infty} \frac{\Gamma\left(\frac{n}{2}+m+\alpha+1\right)}{\Gamma\left(\frac{n}{2}+m\right) \Gamma(\alpha+1)} Z_{m}(x, y) .
$$

Using that $Z_{m}(y, y)=h_{m}|y|^{2 m}$, where $h_{m}$ denotes the dimension of the space $\mathscr{H}_{m}(S)$ (see [1, page 80$]$ ), we get

$$
R_{\alpha}(y, y)=\frac{2}{n V(B)} \sum_{m=0}^{\infty} \frac{\Gamma\left(\frac{n}{2}+m+\alpha+1\right)}{\Gamma\left(\frac{n}{2}+m\right) \Gamma(\alpha+1)} h_{m}|y|^{2 m} .
$$

The numbers $h_{m}$ can be expressed in terms of binomial coefficients:

$$
h_{m}=\left(\begin{array}{c}
n+m-2 \\
n-2
\end{array}\right)+\left(\begin{array}{c}
n+m-3 \\
n-2
\end{array}\right),
$$

for $m \geq 1$ (see [1, page 94]), and it is easily shown that $h_{m} \sim 2 m^{n-2} /(n-2)$ ! as $m \rightarrow \infty$. By Stirling's formula, $\Gamma(m+c) / m ! \sim m^{c-1}$ as $m \rightarrow \infty$, for all $c>0$. Using $\approx$ to denote that the ratio of two quantities is bounded above and below by constants independent of the variable, we thus have $\left(\Gamma\left(\frac{n}{2}+m+\alpha+1\right) / \Gamma\left(\frac{n}{2}+m\right)\right) h_{m} \approx$ $\Gamma(m+n+\alpha) / m !$, and conclude that

$$
R_{\alpha}(y, y) \approx \frac{1}{\left(1-|y|^{2}\right)^{n+\alpha}} .
$$




\section{Compact Toeplitz operators with continuous symbols}

In this section we will describe compactness of Toeplitz operators on the harmonic Bergman spaces $b^{2 . \alpha}(B)$ with uniformly continuous symbols. Our results generalize those recently obtained by Miao [4] for Toeplitz operators on the unweighted harmonic Bergman space $(\alpha=0)$. Miao made use of the explicit formula for the reproducing kernel of the unweighted harmonic Bergman space given in [1, Chapter 8]. For weighted harmonic Bergman spaces no such formula is available, and we will use a different approach to obtain estimates on the reproducing kernels.

PROPOSITION 3.1. Let $-1<\alpha<\infty$. If $f \in L^{\infty}(B)$ has compact support, then $T_{f}$ is compact on $b^{2, \alpha}(B)$.

PROOF. If $f \in L^{\infty}(B)$ is supported in $\rho \bar{B}$, where $0<\rho<1$, then

$$
\int_{B} \int_{B}|f(x)|\left|R_{\alpha}(x, y)\right|^{2} d V(x) d V(y) \leq\|f\|_{\infty} \int_{\rho B} R_{\alpha}(y, y) d V(y)<\infty,
$$

so $T_{f}$ is Hilbert-Schmidt on $b^{2, \alpha}(B)$.

Proposition 3.2. Let $-1<\alpha<\infty$. In $b^{2 . \alpha}(B)$ we have $R_{\alpha}(\cdot, y) /\left\|R_{\alpha}(\cdot, y)\right\| \rightarrow 0$ weakly as $|y| \rightarrow 1^{-}$.

PROOF. Using the reproducing property and (2.7) we have

$$
\left\|R_{\alpha}(\cdot, y)\right\|^{2}=\left\langle R_{\alpha}(\cdot, y), R_{\alpha}(\cdot, y)\right\rangle=R_{\alpha}(y, y) \approx \frac{1}{\left(1-|y|^{2}\right)^{n+\alpha}} .
$$

It is easily verified that $V_{\alpha}\left(B(y,(1-|y|) / 2) \approx(1-|y|)^{n+\alpha}\right.$. So, if $u \in b^{2, \alpha}(B)$, then it follows with the help of (1.2) that

$$
\mid\left\langle u, \frac{R_{\alpha}(\cdot, y)}{\left\|R_{\alpha}(\cdot, y)\right\|}\right\rangle \leq C\left(\int_{B(y,(1-|y|) / 2)}|u(x)|^{2} d V_{\alpha}(x)\right)^{1 / 2},
$$

and thus $\left\langle u, R_{\alpha}(\cdot, y) /\left\|R_{\alpha}(\cdot, y)\right\|\right\rangle \rightarrow 0$ as $|y| \rightarrow 1^{-}$.

For $-1<\alpha<\infty$ and $f \in L^{\infty}(B)$ define the Berezin transform $\tilde{f}$ of $f$ by

$$
\tilde{f}(y)=\left\langle T_{f} \frac{R_{\alpha}(\cdot, y)}{\left\|R_{\alpha}(\cdot, y)\right\|}, \frac{R_{\alpha}(\cdot, y)}{\left\|R_{\alpha}(\cdot, y)\right\|}\right\rangle, \quad y \in B .
$$

Note that even though this is not reflected in the notation, $\tilde{f}$ does depend upon $\alpha$. Clearly, $|\tilde{f}(y)| \leq\left\|T_{f}\right\| \leq\|f\|_{\infty}$, for all $y \in B$. 
If $T_{f}$ is compact on $b^{2 . \alpha}(B)$, then $T_{f}$ maps weakly null sequences to norm null sequences, so that by Proposition 3.2, $\left\|T_{f}\left(R_{\alpha}(\cdot, y) /\left\|R_{\alpha}(\cdot, y)\right\|\right)\right\| \rightarrow 0$ as $|y| \rightarrow 1^{-}$, and thus $\tilde{f}(y) \rightarrow 0$ as $|y| \rightarrow 1^{-}$. We do not know whether the converse is true in general. In the next section we will prove that the converse holds if $f$ is a radial function. Let $C_{0}(B)$ denote the subalgebra of $C(\bar{B})$ consisting of all continuous functions $g$ on $B$ such that $g(y) \rightarrow 0$ as $|y| \rightarrow 1^{-}$. For uniformly continuous symbols we have the following result.

THEOREM 3.3. Let $-1<\alpha<\infty$ and $f \in C(\bar{B})$. Then: $T_{f}$ is compact on $b^{2 . \alpha}(B)$ if and only if $f \in C_{0}(B)$.

In the proof we will need the following lemma.

LEMMA 3.4. Let $-1<\alpha<\infty$. If $0<\delta<1$, then there exists a finite positive number $C_{\delta}$ such that $\left|R_{\alpha}(x, y)\right| \leq C_{\delta}$ whenever $x, y \in B$ are such that $|x-y| \geq \delta$.

PROOF. If $k$ is an integer, then it follows from [2, Lemma 3.2] that there exists a finite number $C$ such that $\left|R_{k}(x, y)\right| \leq C /\left(1-2 x \cdot y+|x|^{2}|y|^{2}\right)^{(n+k) / 2}$, for all $x, y \in B$. Since $1-2 x \cdot y+|x|^{2}|y|^{2}=|x-y|^{2}+\left(1-|x|^{2}\right)\left(1-|y|^{2}\right)>|x-y|^{2}$, we obtain

$$
\left|R_{k}(x, y)\right| \leq \frac{C}{|x-y|^{n+k}},
$$

for all $x, y \in B$, and the stated result follows with $C_{\delta}=C / \delta^{n+k}$.

Suppose $k-1<\alpha<k$, where $k \geq 0$ is an integer. Using (2.5) it is easy to verify that

$$
R_{\alpha}(x, y)=\frac{k !}{\Gamma(\alpha+1) \Gamma(k-\alpha)} \int_{0}^{1} 2 t^{n+2 \alpha+1} R_{k}(t x, t y)\left(1-t^{2}\right)^{k-\alpha-1} d t
$$

If $x, y \in B$ are such that $|x-y| \geq \delta$ it follows from (3.6) and (3.5) that

$$
\begin{aligned}
\left|R_{\alpha}(x, y)\right| & \leq \frac{k !}{\Gamma(\alpha+1) \Gamma(k-\alpha)} \int_{0}^{1} 2 t^{n+2 \alpha+1}\left|R_{k}(t x, t y)\right|\left(1-t^{2}\right)^{k-\alpha-1} d t \\
& \leq \frac{k !}{\Gamma(\alpha+1) \Gamma(k-\alpha)} \frac{2 C}{\delta^{n+k}} \int_{0}^{1} t^{2 \alpha-k+1}\left(1-t^{2}\right)^{k-\alpha-1} d t .
\end{aligned}
$$

So the constant $C_{\delta}=C \Gamma\left(\alpha-\frac{k}{2}+1\right) k ! /\left(\delta^{n+k} \Gamma(\alpha+1) \Gamma\left(\frac{k}{2}+1\right)\right)$ works.

PROOF OF THEOREM 3.3. The implication using $f \in C_{0}(B)$ follows easily from Proposition 3.1. 
To prove the other implication we need only show that $\tilde{f}-f \in C_{0}(B)$. Using Lemma 3.4 and the observation that

$$
\tilde{f}(y)-f(y)=\frac{1}{R_{\alpha}(y, y)} \int_{B}(f(x)-f(y)) R_{\alpha}(x, y)^{2} d V_{\alpha}(x)
$$

we get

$$
\begin{aligned}
|\tilde{f}(y)-f(y)| & \leq \frac{1}{R_{\alpha}(y, y)} \int_{B}|f(x)-f(y)| R_{\alpha}(x, y)^{2} d V_{\alpha}(x) \\
& \leq \omega(\delta) \frac{1}{R_{\alpha}(y, y)} \int_{B(y, \delta)} R_{\alpha}(x, y)^{2} d V_{\alpha}(x)+\frac{2 C_{\delta}^{2}\|f\|_{\infty}}{R_{\alpha}(y, y)} \\
& \leq \omega(\delta)+C_{\delta}^{\prime}\left(1-|y|^{2}\right)^{n+\alpha}\|f\|_{\infty},
\end{aligned}
$$

where $\omega(\delta)=\sup \{|f(x)-f(z)|: x, z \in B,|x-z|<\delta\}$. Letting $|y| \rightarrow 1^{-}$we obtain

$$
\limsup _{|y| \rightarrow 1^{-}}|\tilde{f}(y)-f(y)| \leq \omega(\delta),
$$

for each $0<\delta<1$. Since $f \in C(\bar{B}), \omega(\delta) \rightarrow 0$ as $\delta \rightarrow 0^{+}$we conclude that $\tilde{f}(y)-f(y) \rightarrow 0$ as $|y| \rightarrow 1^{-}$.

If $\mathscr{L}\left(b^{2 . \alpha}(B)\right)$ denotes the Banach algebra of all bounded linear operators on $b^{2 . \alpha}(B)$, and $\mathscr{K}$ denotes the ideal of compact operators in $\mathscr{L}\left(b^{2 . \alpha}(B)\right)$, then the essential spectrum of an operator $T$ in $\mathscr{L}\left(b^{2 . \alpha}(B)\right)$, denoted by $\sigma_{e}(T)$, is the spectrum of the operator $T+\mathscr{K}$ in the Calkin algebra $\mathscr{L}\left(b^{2 . \alpha}(B)\right) / \mathscr{K}$.

THEOREM 3.7. Let $-1<\alpha<\infty$ and $f \in C(\bar{B})$. The essential spectrum of the operator $T_{f}$ on $b^{2 . \alpha}(B)$ is $\sigma_{c}\left(T_{f}\right)=f(S)$.

In the proof of the above theorem we will make use of Hankel operators. For $f \in L^{\infty}(B)$ the Hankel operator $H_{f}: b^{2 . \alpha}(B) \rightarrow L^{2}\left(B, d V_{\alpha}\right)$ is defined by $H_{f} u=$ $\left(I-Q_{\alpha}\right)[f u], u \in b^{2 . \alpha}(B)$. In [5] it was shown that for every $f \in C(\bar{B})$ the Hankel operator $H_{f}$ is compact on $b^{2 . \alpha}(B)$. The following identity gives a simple relationship between Toeplitz and Hankel operators:

$$
T_{f g}-T_{f} T_{g}=H_{f}^{*} H_{g},
$$

for $f, g \in L^{\infty}(B)$.

Proof OF THEOREM 3.7. We first show that $f(S) \subset \sigma_{e}\left(T_{f}\right)$. Suppose $\xi=f(\zeta)$, with $\zeta \in S$. We claim that $T_{f-\xi}$ cannot be left-invertible in the Calkin algebra, so that $\xi \in \sigma_{e}\left(T_{f}\right)$. To prove this claim we first observe that the argument in the proof of 
Theorem 3.3 shows that $\left\|(f-f(y)) R_{\alpha}(\cdot, y) /\right\| R_{\alpha}(\cdot, y)\|\| \rightarrow 0$ as $|y| \rightarrow 1^{-}$, and thus $\left\|T_{f-\xi} R_{\alpha}(\cdot, y) /\right\| R_{\alpha}(\cdot, y)\|\| \rightarrow 0$ as $y \rightarrow \zeta$ in $B$. If $T$ is a bounded linear operator on $b^{2, \alpha}(B)$, then

$$
\left\langle\left(I-T T_{f-\xi}\right) \frac{R_{\alpha}(\cdot, y)}{\left\|R_{\alpha}(\cdot, y)\right\|}, \frac{R_{\alpha}(\cdot, y)}{\left\|R_{\alpha}(\cdot, y)\right\|}\right\rangle=1-\left\langle T_{f-\xi} \frac{R_{\alpha}(\cdot, y)}{\left\|R_{\alpha}(\cdot, y)\right\|}, \frac{T^{*} R_{\alpha}(\cdot, y)}{\left\|R_{\alpha}(\cdot, y)\right\|}\right\rangle \rightarrow 1
$$

as $y \rightarrow \zeta$ in $B$. It follows that $T T_{f-\xi}-I$ cannot be compact on $b^{2, \alpha}(B)$, proving our claim.

To complete the proof we show that if $\xi \in \mathbb{C} \backslash f(S)$, then $T_{f-\xi}$ is invertible in the Calkin algebra. Suppose $\xi \notin f(S)$. Then there are $0<r<1$ and $g \in C(\bar{B})$ such that $(f-\xi) g=1$ on $\bar{B} \backslash B(0, r)$. The function $h=1-(f-\xi) g$ is compactly supported, so by Proposition 3.1, $T_{h}$ is compact on $b^{2 . \alpha}(B)$. Using (3.8) we have

$$
T_{f-\xi} T_{g}=T_{(f-\xi) g}-H_{\bar{f}-\bar{\xi}}^{*} H_{g}=I-T_{h}-H_{\tilde{f}}^{*} H_{g} .
$$

By [5, Theorem 4.3], the operator $H_{f}^{*} H_{g}$ is compact, thus $T_{h}+H_{f}^{*} H_{g} \in \mathscr{K}$, and consequently $T_{f-\xi}$ is right-invertible in the Calkin algebra. That $T_{f-\xi}$ is also leftinvertible in the Calkin algebra is proved similarly.

COROLLARY 3.9. Let $-1<\alpha<\infty$. If $f \in C(\bar{B})$, then the essential norm of $T_{f}$ on $b^{2 . \alpha}(B)$ is given by $\left\|T_{f}\right\|_{e}=\sup _{\zeta \in S}|f(\zeta)|$.

PROOF. By (3.8), $T_{f}^{*} T_{f}-T_{f} T_{f}^{*}=H_{\bar{f}}^{*} H_{\bar{f}}-H_{f}^{*} H_{f}$ is compact on $b^{2 . \alpha}(B)$. Thus $T_{f}+\mathscr{K}$ is normal in the Calkin algebra, so that its norm is equal to its spectral radius, and the stated result follows from Theorem 3.7.

\section{Compact Toeplitz operators with radial symbols}

Korenblum and Zhu [3] proved that for a radial symbol the Toeplitz operator on the Bergman space of analytic functions on the unit disk is compact precisely when its Berezin transform vanishes near the unit circle. In [6] the author generalized Korenblum and Zhu's result to the setting of weighted Bergman spaces of analytic functions on the unit ball in $\mathbb{C}^{n}$. The following theorem shows that the analogous result holds for Toeplitz operators on weighted harmonic Bergman spaces.

THEOREM 4.1. Let $-1<\alpha<\infty$ and let $f$ be a bounded measurable radial function on $B$. Then: $T_{f}$ is compact on $b^{2 \cdot \alpha}(B)$ if and only if $\tilde{f} \in C_{0}(B)$.

The proof of Theorem 4.1 makes use of the following lemma. 
LEMMA 4.2. If $f$ is a bounded measurable radial function on $B$, then each homogeneous harmonic polynomial of degree $m$ is an eigenvector of $T_{f}$ with eigenvalue given by

$$
\lambda_{m}=\frac{\Gamma\left(\frac{n}{2}+m+\alpha+1\right)}{\Gamma\left(\frac{n}{2}+m\right) \Gamma(\alpha+1)} \int_{0}^{1} 2 r^{n+2 m-1} \varphi(r)\left(1-r^{2}\right)^{\alpha} d r,
$$

where $\varphi$ is a bounded measurable function on $[0,1)$, for which $f(x)=\varphi(|x|)$, for all $x \in B$.

PROOF. If $p$ is an $m$-homogeneous harmonic polynomial, then, using (2.3), (2.4) and (2.5),

$$
\int_{S} p(\zeta) R_{\alpha}(r \zeta, y) d \sigma(\zeta)=\frac{2 \Gamma\left(\frac{n}{2}+m+\alpha+1\right)}{n V(B) \Gamma\left(\frac{n}{2}+m\right) \Gamma(\alpha+1)} r^{m} p(y)
$$

and thus we have

$$
\begin{aligned}
\left(T_{f} p\right)(y) & =\int_{B} f(x) p(x) R_{\alpha}(x, y) d V_{\alpha}(x) \\
& =n V(B) \int_{0}^{1} r^{n+m-1} \varphi(r) \int_{S} p(\zeta) R_{\alpha}(r \zeta, y) d \sigma(\zeta)\left(1-r^{2}\right)^{\alpha} d r \\
& =p(y) \frac{\Gamma\left(\frac{n}{2}+m+\alpha+1\right)}{\Gamma\left(\frac{n}{2}+m\right) \Gamma(\alpha+1)} \int_{0}^{1} 2 r^{n+2 m-1} \varphi(r)\left(1-r^{2}\right)^{\alpha} d r,
\end{aligned}
$$

establishing the proof.

As in Korenblum and Zhu's argument, we will need a Tauberian theorem. The following lemma follows from a classical result of Hardy and Littlewood (see [6]).

LEMMA 4.4. Let $0<v<\infty$, and let $\left(b_{m}\right)$ be a sequence of complex numbers for which $\sup \left\{\left|(m+1) b_{m}-m b_{m-1}\right|: m>1\right\}<\infty$. Then:

$$
(1-t)^{v+1} \sum_{m=0}^{\infty} \frac{\Gamma(m+v+1)}{m ! \Gamma(v+1)} b_{m} t^{m} \rightarrow 0 \text { as } t \rightarrow 1^{-}
$$

if and only if $b_{m} \rightarrow 0$ as $m \rightarrow \infty$.

PROOF OF THEOREM 4.1. If $f(x)=\varphi(|x|)$, for all $x \in B$, where $\varphi$ is a bounded measurable function on $[0,1)$, then integrating by polar coordinates, using $(2.5)$ and (2.4), it is readily verified that

$$
\tilde{f}(y)=\frac{1}{R_{\alpha}(y, y)} \sum_{m=0}^{\infty} \frac{\Gamma\left(\frac{n}{2}+m+\alpha+1\right)}{\Gamma\left(\frac{n}{2}+m\right) \Gamma(\alpha+1)} h_{m} \lambda_{m}|y|^{2 m},
$$


where $\lambda_{m}$ is as in (4.3).

Now, suppose $\tilde{f}(y) \rightarrow 0$ as $|y| \rightarrow 1^{-}$. Using (2.7) we get

$$
\left(1-|y|^{2}\right)^{n+\alpha} \sum_{m=0}^{\infty} \frac{\Gamma\left(\frac{n}{2}+m+\alpha+1\right)}{\Gamma\left(\frac{n}{2}+m\right) \Gamma(\alpha+1)} h_{m} \lambda_{m}|y|^{2 m} \rightarrow 0
$$

as $|y| \rightarrow 1^{-}$. We will use Lemma 4.4 to prove that $\lambda_{m} \rightarrow 0$ as $m \rightarrow \infty$. We first show that $\sup \left\{m\left|\lambda_{m}-\lambda_{m-1}\right|: m \geq 1\right\}<\infty$. Rewriting the integrand in the integral in (4.3) using $r^{2} \varphi(r)=\varphi(r)-\varphi(r)\left(1-r^{2}\right)$, it is easily seen that

$$
\lambda_{m}=\frac{n+2 m+2 \alpha}{n+2 m-2} \lambda_{m-1}+\frac{\Gamma\left(\frac{n}{2}+m+\alpha+1\right)}{\Gamma\left(\frac{n}{2}+m\right) \Gamma(\alpha+1)} \int_{0}^{1} 2 r^{n+2 m-3} \varphi(r)\left(1-r^{2}\right)^{\alpha+1} d r,
$$

thus

$$
\begin{aligned}
\lambda_{m}- & \lambda_{m-1} \\
& =\frac{2(1+\alpha)}{n+2 m-2} \lambda_{m-1}+\frac{\Gamma\left(\frac{n}{2}+m+\alpha+1\right)}{\Gamma\left(\frac{n}{2}+m\right) \Gamma(\alpha+1)} \int_{0}^{1} 2 r^{n-2 m-3} \varphi(r)\left(1-r^{2}\right)^{\alpha+1} d r,
\end{aligned}
$$

and the claim follows from the estimate

$$
\begin{aligned}
\left|\int_{0}^{1} 2 r^{n+2 n-3} \varphi(r)\left(1-r^{2}\right)^{\alpha+1} d r\right| & \leq\|\varphi\|_{\infty} \int_{0}^{1} 2 r^{n-2 m-3}\left(1-r^{2}\right)^{\alpha+1} d r \\
& =\|f\|_{\infty} \frac{\Gamma\left(\frac{n}{2}+m-1\right) \Gamma(\alpha+2)}{\Gamma\left(\frac{n}{2}+m+\alpha+1\right)} .
\end{aligned}
$$

Write

$$
\frac{\Gamma\left(\frac{n}{2}+m+\alpha+1\right)}{\Gamma\left(\frac{n}{2}+m\right) \Gamma(\alpha+1)} h_{m} \lambda_{m}=\frac{\Gamma(m+n+\alpha)}{m ! \Gamma(n+\alpha)} b_{m} .
$$

Then

$$
(m+1) b_{m}-m b_{m-1}=\left\{(m+1) a_{m}-m a_{m-1}\right\} \lambda_{m}+m\left(\lambda_{m}-\lambda_{m-1}\right) a_{m-1},
$$

where the $a_{n}$ are given by

$$
a_{m}=\frac{m ! \Gamma(n+\alpha)}{\Gamma(m+n+\alpha)} \frac{\Gamma\left(\frac{n}{2}+m+\alpha+1\right)}{\Gamma\left(\frac{n}{2}+m\right) \Gamma(\alpha+1)} h_{m} .
$$

It follows that the $b_{m}$ satisfy the condition of Lemma 4.4 once we show that the $(m+1) a_{m}-m a_{m-1}$ are bounded. A calculation shows that

$$
\begin{aligned}
& (m+1) a_{m}-m a_{m-1}=\frac{(m+1) ! \Gamma(n+\alpha) \Gamma\left(\frac{n}{2}+m+\alpha+1\right)}{\Gamma(m+n+\alpha) \Gamma\left(\frac{n}{2}+m\right) \Gamma(\alpha+1)}\left(h_{m}-h_{m-1}\right) \\
& +\frac{(3-n) m+(n+\alpha)\left(2-\frac{n}{2}\right)-1}{(m+n+\alpha-1)\left(\frac{n}{2}+m-1\right)} \frac{m ! \Gamma(n+\alpha) \Gamma\left(\frac{n}{2}+m+\alpha\right)}{\Gamma(m+n+\alpha-1) \Gamma\left(\frac{n}{2}+m-1\right) \Gamma(\alpha+1)} h_{m-1} .
\end{aligned}
$$


It follows easily from (2.6) that

$$
h_{m}-h_{m-1}=\frac{(n+2 m-2)(n-1)}{m(m-1)} \frac{(n+m-4) !}{(m-2) !(n-2) !},
$$

for $m \geq 2$, thus $h_{m}-h_{m-1} \approx m^{n-3}$. Recalling that $h_{m} \approx m^{m-2}$, our claim that the $(m+1) a_{m}-m a_{m-1}$ are bounded follows with the help of Stirling's formula. Applying Lemma 4.4 we conclude that $b_{m} \rightarrow 0$ as $m \rightarrow \infty$. Since the $a_{m}$ have a non-zero limit, we conclude that $\lambda_{m} \rightarrow 0$ as $m \rightarrow \infty$, and thus $T_{f}$ is compact on $b^{2 \cdot \alpha}(\underline{B})$.

\section{Compact Toeplitz operators with discontinuous symbols}

We write $b^{2}(B)$ for the unweighted harmonic Bergman space $b^{2.0}(B)$. If $T_{f}$ is compact on $b^{2}(B)$, then $T_{f}$ : need not be compact. In fact, there are functions $f$ on $B$ for which $T_{f}$ is compact, and $T_{f^{2}}$ is the identity operator on $b^{2}(B)$. We will show this by considering a class of Toeplitz operators whose symbols are radial functions taking only the values 1 and -1 .

Let $\left(r_{k}\right)$ and $\left(s_{k}\right)$ be sequences of positive numbers converging to 1 with $0=r_{1}<$ $s_{1}<r_{2}<s_{2}<\cdots$, and define $f$ on $B$ by

$$
f(x)= \begin{cases}-1, & \text { if } r_{k} \leq|x|<s_{k} \\ 1, & \text { if } s_{k} \leq|x|<r_{k+1}\end{cases}
$$

By Lemma 4.2 the eigenvalues of $T_{f}$ are given by

$$
\lambda_{m}=\sum_{k=1}^{\infty}\left(r_{k+1}^{n+2 m}-2 s_{k}^{n+2 m}+r_{k}^{n+2 m}\right)
$$

THEOREM 5.3. Let $f$ be the function given by (5.1) with $r_{k}=1-1 / k^{c}$, where $c>0$, and $s_{k}=\left(r_{k}+r_{k+1}\right) / 2$. Then: $T_{f}$ is trace-class on $b^{2}(B)$ if $c<1 /(n-1)$.

PROOF. Note that each of the terms of the series in (5.2) is positive. Using that the multiplicity of $\lambda_{m}$ is $h_{m}$ and the fact that $h_{m} \approx(m+n-2) ! / m !$, we conclude that

$$
\begin{aligned}
\operatorname{tr}\left(T_{f}\right)=\sum_{m=0}^{\infty} \lambda_{m} h_{m} & =\sum_{k=1}^{\infty} \sum_{m=0}^{\infty}\left\{r_{k+1}^{n+2 m}+r_{k}^{n+2 m}-2 s_{k}^{n+2 m}\right\} h_{m} \\
& \leq C \sum_{k=1}^{\infty} \sum_{m=0}^{\infty}\left\{r_{k+1}^{n+2 m}+r_{k}^{n+2 m}-2 s_{k}^{n+2 m}\right\} \frac{(m+n-2) !}{m !(n-2) !} \\
& =C \sum_{k=1}^{\infty}\left\{\frac{r_{k+1}^{n}}{\left(1-r_{k+1}^{2}\right)^{n-1}}+\frac{r_{k}^{n}}{\left(1-r_{k}^{2}\right)^{n-1}}-\frac{2 s_{k}^{n}}{\left(1-s_{k}^{2}\right)^{n-1}}\right\} \\
& \leq C^{\prime} \sum_{k=1}^{\infty} \frac{\delta_{k}^{2}}{\left(1-r_{k}\right)^{n+1}},
\end{aligned}
$$


where $\delta_{k}=r_{k+1}-s_{k}=s_{k}-r_{k}$. So, if $\sum_{k=1}^{\infty} \delta_{k}^{2} /\left(1-r_{k}\right)^{n+1}<\infty$ then $T_{f}$ is trace-class on $b^{2}(B)$. For $r_{k}=1-1 / k^{c}$ and $s_{k}=\left(r_{k}+r_{k+1}\right) / 2$ we have $2 \delta_{k}=1 / k^{c}-1 /(k+1)^{c} \approx$ $1 / k^{c+1}$, thus $\delta_{k}^{2} /\left(1-r_{k}\right)^{n+1} \approx 1 / k^{2-(n-1) c}$, and we see that $T_{f}$ is trace-class on $b^{2}(B)$ if $(n-1) c<1$, that is, if $c<1 /(n-1)$.

THEOREM 5.4. Let $f$ be the function given by (5.1) with $s_{k}=\left(r_{k}+r_{k+1}\right) / 2$ and put $\delta_{k}=r_{k+1}-s_{k}=s_{k}-r_{k}$. Then: $T_{f}$ is compact on $b^{2}(B)$ if and only if

$$
\delta_{k} /\left(1-r_{k}\right) \rightarrow 0 \text { as } k \rightarrow \infty
$$

In the proof of the above theorem we will need the following lemma.

LEMMA 5.5. Let $m$ be a positive integer, and let $\left(r_{k}\right)$ be an increasing sequence of positive numbers converging to 1 and $2 \delta_{k}=r_{k+1}-r_{k}$, for all $k$, then

$$
\sum_{k=1}^{\infty} \frac{2 \delta_{k}}{\left(1-r_{k}^{2} t\right)^{m+1}} \leq \frac{1}{(1-t)^{m}}
$$

for all $0<t<1$.

PROOF. Since $r_{k+1}>r_{k}$, we have

$$
r_{k+1}^{j+1}-r_{k}^{j+1} \geq\left(r_{k+1}-r_{k}\right)(j+1) r_{k}^{j} \geq 2(j+1) \delta_{k} r_{k}^{2 j},
$$

and consequently

$$
\sum_{k=1}^{\infty} 2 \delta_{k} r_{k}^{2 j} \leq \frac{1}{j+1} \sum_{k=1}^{\infty}\left(r_{k+1}^{j+1}-r_{k}^{j+1}\right)=\frac{1}{j+1}\left(1-r_{1}^{j+1}\right) \leq \frac{1}{j+1} .
$$

Thus

$$
\begin{aligned}
\sum_{k=1}^{\infty} \frac{2 \delta_{k}}{\left(1-r_{k}^{2} t\right)^{m+1}} & =\sum_{j=0}^{\infty} \frac{(j+m) !}{j ! m !}\left\{\sum_{k=1}^{\infty} 2 \delta_{k} r_{k}^{2 j}\right\} t^{j} \leq \sum_{j=0}^{\infty} \frac{(j+m) !}{j ! m !} \frac{1}{j+1} t^{j} \\
& =\frac{1}{m}\left\{\frac{1}{\left(1-|t|^{2}\right)^{m}}-1\right\} / t \leq \frac{1}{(1-t)^{m}},
\end{aligned}
$$

proving the stated result.

PROOF OF THEOREM 5.4. Write $R$ for the reproducing kernel of $b^{2}(B)$.

Step 1. Using equation (2.5) it is easy to show that for $0<r<1$ we have

$$
\int_{r B} R(x, y)^{2} d V(x)=\sum_{m=0}^{\infty}(n+2 m) r^{n+2 m} h_{m}|y|^{2 m} .
$$


Consequently,

$$
\tilde{f}(y)=\frac{1}{R(y, y)} \sum_{k=1}^{\infty} \sum_{m=0}^{\infty}(n+2 m)\left(r_{k+1}^{n+2 m}-2 s_{k}^{n+2 m}+r_{k}^{n+2 m}\right) h_{m}|y|^{2 m} .
$$

Step 2. Similar to the proof of Theorem 5.3: there exists a finite positive constant $C$ such that

$$
\tilde{f}(y) \leq C\left(1-|y|^{2}\right)^{n} \sum_{k=1}^{\infty}\left\{\frac{r_{k+1}^{n}}{\left(1-r_{k+1}^{2}|y|^{2}\right)^{n}}+\frac{r_{k}^{n}}{\left(1-r_{k}^{2}|y|^{2}\right)^{n}}-\frac{2 s_{k}^{n}}{\left(1-s_{k}^{2}|y|^{2}\right)^{n}}\right\},
$$

for all $y \in B$.

Step 3 . There is a finite positive constant $M$ for which we have the inequality

$$
\frac{r_{k+1}^{n}}{\left(1-r_{k+1}^{2}|y|^{2}\right)^{n}}+\frac{r_{k}^{n}}{\left(1-r_{k}^{2}|y|^{2}\right)^{n}}-\frac{2 s_{k}^{n}}{\left(1-s_{k}^{2}|y|^{2}\right)^{n}} \leq \frac{M \delta_{k}^{2}}{\left(1-r_{k}^{2}|y|^{2}\right)^{n+2}},
$$

for all $k \geq 1$ and all $y \in B$.

Step 4. It follows from steps 2 and 3 and Lemma 5.5 that

$$
\begin{aligned}
\tilde{f}(y) \leq & C\left(1-|y|^{2}\right)^{n} \sum_{k=1}^{K}\left\{\frac{r_{k+1}^{n}}{\left(1-r_{k+1}^{2}|y|^{2}\right)^{n}}+\frac{r_{k}^{n}}{\left(1-r_{k}^{2}|y|^{2}\right)^{n}}-\frac{2 s_{k}^{n}}{\left(1-s_{k}^{2}|y|^{2}\right)^{n}}\right\} \\
& +C^{\prime}\left(1-|y|^{2}\right)^{n} \sum_{k=K}^{\infty} \frac{\delta_{k}^{2}}{\left(1-r_{k}^{2}|y|^{2}\right)^{n+2}} \\
\leq & C\left(1-|y|^{2}\right)^{n} \sum_{k=1}^{K}\left\{\frac{r_{k+1}^{n}}{\left(1-r_{k+1}^{2}|y|^{2}\right)^{n}}+\frac{r_{k}^{n}}{\left(1-r_{k}^{2}|y|^{2}\right)^{n}}-\frac{2 s_{k}^{n}}{\left(1-s_{k}^{2}|y|^{2}\right)^{n}}\right\} \\
& +C^{\prime}\left(1-|y|^{2}\right)^{n} \sup _{k \geq K}\left(\frac{\delta_{k}}{1-r_{k}}\right) \sum_{k=K}^{\infty} \frac{\delta_{k}}{\left(1-r_{k}^{2}|y|^{2}\right)^{n+1}} \\
\leq & C\left(1-|y|^{2}\right)^{n} \sum_{k=1}^{K}\left\{\frac{r_{k+1}^{n}}{\left(1-r_{k+1}^{2}|y|^{2}\right)^{n}}+\frac{r_{k}^{n}}{\left(1-r_{k}^{2}|y|^{2}\right)^{n}}-\frac{2 s_{k}^{n}}{\left(1-s_{k}^{2}|y|^{2}\right)^{n}}\right\} \\
& +C^{\prime} \sup _{k \geq K}\left(\frac{\delta_{k}}{1-r_{k}}\right),
\end{aligned}
$$

for all $y \in B$ and every integer $K \geq 1$. Because each of the terms in the series (5.7) is positive,

$$
\limsup _{|y| \rightarrow 1^{-}}|\tilde{f}(y)| \leq C^{\prime} \sup _{k \geq K}\left(\frac{\delta_{k}}{1-r_{k}}\right),
$$

for every integer $K \geq 1$. So, if $\delta_{k} /\left(1-r_{k}\right) \rightarrow 0$ as $k \rightarrow \infty$, then $\tilde{f}(y) \rightarrow 0$ as $|y| \rightarrow 1^{-}$, and by Theorem $4.1, T_{f}$ is compact on $b^{2}(B)$. 
To prove the converse, note that the inequalities in steps 2 and 3 can be reversed to obtain

$$
\tilde{f}(y) \geq c\left(1-|y|^{2}\right)^{n} \sum_{k=1}^{\infty} \frac{\delta_{k}^{2}}{\left(1-r_{k}^{2}|y|^{2}\right)^{n+2}},
$$

for all $y \in B$. In particular, for $\zeta \in S$ we have $\tilde{f}\left(r_{k} \zeta\right) \geq c\left(1-r_{k}^{2}\right)^{n} \delta_{k}^{2} /\left(1-r_{k}^{4}\right)^{n+2}$, which easily implies that $\left(\delta_{k} /\left(1-r_{k}\right)\right)^{2} \leq C \tilde{f}\left(r_{k} \zeta\right)$, for all $k \geq 1$. If $T_{f}$ is compact on $b^{2}(B)$, then $\tilde{f}\left(r_{k} \zeta\right) \rightarrow 0$, and hence $\delta_{k} /\left(1-r_{k}\right) \rightarrow 0$ as $k \rightarrow \infty$.

COROLLARY 5.8. Let $f$ be the function given by (5.1) with $r_{k}=1-1 / k^{c}$, where $c>0$ and $s_{k}=\left(r_{k}+r_{k+1}\right) / 2$. Then $T_{f}$ is compact on $b^{2}(B)$.

PROOF. Since $\delta_{k} \approx 1 / k^{c+1}$, we have $\delta_{k} /\left(1-r_{k}\right) \approx 1 / k$.

\section{Acknowledgements}

The research for this paper was mostly done while visiting the Free University, Amsterdam, The Netherlands, on sabbatical leave; I thank the University of Montana for awarding me a sabbatical and the Mathematics Department of the Free University for its hospitality and support.

\section{References}

[1] S. Axler, P. Bourdon, and W. Ramey, Harmonic function theory (Springer, New York, 1992).

[2] R. Coifman and R. Rochberg, 'Representation theorems for holomorphic and harmonic functions,' Astérisque 77 (1980), 11-66.

[3] B. Korenblum and K. Zhu, 'An application of Tauberian theorems to Toeplitz operators,' J. Operator Theory 33 (1995), 353-361.

[4] J. Miao, 'Toeplitz operators on harmonic Bergman spaces,' Integral Equations Operator Theory 27 (1997), 426-438.

[5] K. Stroethoff, 'Compact Hankel operators on weighted harmonic Bergman spaces,' Glasgow' Math. J. 39 (1997), 77-84.

[6] K. Stroethoff, 'Compact Toeplitz operators on Bergman spaces,' Math. Proc. Cambridge Phil. Soc., to appear.

Department of Mathematical Sciences

University of Montana

Missoula, MT 59812-1032

USA

e-mail:ma_kms@selway.umt.edu 